\title{
Dynamics of microparticles in vacuum breakdown: Cranberg's scenario updated by numerical modeling
}

\author{
B. Seznec, ${ }^{1,2}$ Ph. Dessante, ${ }^{2}$ T. Jager, ${ }^{1}$ L. Caillault, ${ }^{1}$ Ph. Teste, ${ }^{2}$ and T. Minea ${ }^{1}$ \\ ${ }^{1}$ LPGP, Laboratoire de Physique des Gaz et Plasmas, UMR 8578, CNRS, Université Paris-Sud, \\ Université Paris-Saclay, Orsay Cedex 91405, France \\ ${ }^{2}$ GeePs, Group of Electrical Engineering-Paris, UMR 8507, CNRS, Centrale Supelec, \\ Université Paris-Sud, Sorbonne Universités, UPMC Université Paris 06, Université Paris-Saclay, \\ 11 rue Joliot Curie, Plateau de Moulon 91192 Gif sur Yvette Cedex, France
}

(Received 4 August 2016; published 24 July 2017)

\begin{abstract}
Microparticles (MP) and thermofield emission in vacuum are mainly caused by the roughness present at the surface of electrodes holding a high voltage. They can act as a trigger for breakdown, especially under high vacuum. This theoretical study discusses the interactions between one MP and the thermofield emission electron current as well as the consequences on the MP's transit. Starting from Cranberg's assumptions, new phenomena have been taken into account such as MP charge variation due to the secondary electron emission induced by energetic electron bombardment. Hence, the present model can be solved only numerically. Four scenarios have been identified based on the results, depending on the electron emission current from the cathode roughness (tip) and the size of the MP released at the anode, namely (i) one way; (ii) back and forth; (iii) oscillation; and (iv) vaporization. A crash study of the MP on the cathode shows that the electron emission can decrease if the MP covers the thermoemissive tip, i.e., if the MP is larger than the tip size — a phenomenon often called "conditioning" — and helping to increase the voltage holding in vacuum without breakdown.
\end{abstract}

DOI: 10.1103/PhysRevAccelBeams.20.073501

\section{INTRODUCTION}

The maintaining of high voltages under vacuum is still very demanded by the development of several devices and its improvement passes by the understanding of undergoing phenomena that can lead to breakdown. This knowledge is very important in the design and the safe operation of large instruments such as particle accelerators, fusion devices, fast vacuum switches, etc.

For instance, in future magnetically confined fusion reactors (ITER, DEMO), the initiation and the sustainment of fusion reactions require energetic heavy particles [1]. An efficient way to transfer energy and momentum to heavy species of plasmas in fusion uses high power beams of neutral $\mathrm{D}^{\circ}$ atoms at high energy (2 times $35 \mathrm{MW}$ of $\mathrm{D}^{\circ}$ for ITER and even more for DEMO). The injection system called the neutral beam injector presents scientific and technical challenges. For instance, energetic $\mathrm{D}^{\circ}$ are produced by striping reactions from $\mathrm{D}^{-}$ions which are first produced, then accelerated $(1 \mathrm{MeV})$.

To reach such high energies, the negative ions $\mathrm{D}^{-}$are accelerated in a multigrid direct current (dc) system under vacuum [1-3]. A possible limitation of the system could come from the unpredictable breakdown events between

Published by the American Physical Society under the terms of the Creative Commons Attribution 3.0 License. Further distribution of this work must maintain attribution to the author(s) and the published article's title, journal citation, and DOI. grid electrodes or inside the high voltage vacuum feedthrough system called "bushing" [1].

The formation of these discharges in vacuum has been studied over the past few decades [4-6] and several mechanisms have been proposed trying to explain these events.

For small interelectrode distances $(<1 \mathrm{~mm})$, the breakdown voltage varies linearly with the electrode gap [7-9]. This can be explained by the roughness at the cathode surface. If the roughness is assumed to be dominated by the highest microscopic tip, the electric field is localized at the apex of this tip, where initially the cold (i.e., field-effect) electron emission occurs. This current flowing along the microtip leads to its heating by the Joule effect, while the Nottingham effect [10] can either heat or cool down its apex. However, the temperature always increases along the tip, facilitating the electron emission by the addition of a second mechanism, the thermoionic effect. If the tip temperature reaches the vaporization temperature, metal vapors are released above the tip, which expands adiabatically under the vacuum. Hence, locally the pressure increases being supplied by the cathode element vapors. The free electrons released due to the combined thermofield emission can efficiently ionize this vapor [11]. Formed ions are backattracted and they bombard the tip surface emitting more secondary electrons [12]. This scenario is highly nonlinear and the thermal instability cannot be stopped evolving towards the well-known Townsend avalanche, which may be sufficient to initiate the breakdown. Some authors $[13,14]$ 
have shown that the surface of the cathode could be altered under the action of the electric field. Indeed Descoeudres et al. [13] showed an increase of the enhancement coefficient of the electric field at the surface of the cathode before the breakdown and supposed that a small protrusion is pulled out from the surface under the effect of the electrostatic force induced by the field. A possible explanation could be the alignment of an oblique tip under the high electric field, as observed for instance for carbon nanotubes [15]. However, this increase of the enhancement factor of the tip could be overestimated if only the cold emission, i.e., the FowlerNordheim formula, is considered [16]. At low temperature it describes well the emitted electron current, but with the temperature increase at the tip surface, the thermofield emission should govern the electron emission instead of a pure field emission, before the breakdown occurs.

For larger interelectrode distances, experimental results have shown that the breakdown voltage follows a power law regarding the electrode gap distance $(d)$, with the superscript lying between 0.5 and $0.7[17,18]$. Several mechanisms have attempted to explain this saturation of the breakdown voltage for large interelectrode distances such as, for instance, the heating of the anode [19]. The most probable assumption of the origin of this saturation is the presence of microparticles (denoted hereafter MPs) [20-22]. Cranberg has shown analytically that the breakdown voltage, due to the presence of MPs formed at the anode and accelerated in the space between electrodes, obeys as the power law versus the gap as $d^{0.5}$ [18]. Subsequently, Slivkov corrected Cranberg's results proposing the scaling law with $d^{0.63}$ [23]. These assumptions have been confirmed experimentally by the detection of clumps [24,25]. Thus, Cranberg's model is called sometimes "clumps theory" [4].

Accordingly, these MPs can cause the breakdown if they are completely vaporized due to their interaction with energetic electrons originating from a cathode roughness [26] or if they evaporate when crashing one of the electrodes $[18,21]$. In these two cases, the vapor produced by the MP evaporation can be easily ionized and the above described scenario can thus occur. Another possibility is indirectly related to the presence of the MP that can generate a microdischarge when it approaches very close to the tip [27] by locally enhancing the electric field to the surface roughness. This effect can boost the thermofield instability.

Microparticles have been observed also in accelerators. In dc photoinjectors, for instance, MPs or ions of the residual gases [28] can damage the surface and reduce the quantum efficiency of the photocathode. In the storage rings, the MPs can interact with the beam and consequently the lifetime of the beam drastically drops [29-31]. In the Large Hadron Collider (LHC), these beam losses could lead to the quenching of the superconducting magnets.

In this study, the effect of MPs on the breakdown is limited to the dc case because the period of the rf signal is lower than the flight time of the MP in the interelectrode gap, typically $\sim 20 \mu$ s [32].

Several authors treated the dynamics of MPs in the interelectrode gap and described the evolution of MP properties (mass, charge, position) during the flight from the anode towards the cathode $[4,33]$. The aim of this work is to update the former models making them more realistic in order to improve the understanding of involved phenomena in the high voltage breakdown with small and large interelectrode gaps. We present here the numerical analysis of the dynamics of a clump which interacts with electrons emitted from a given cathode roughness, hereafter referred to as tip. The phenomenological model is introduced in the next section. The description of the model background is detailed in the third section considering (i) thermofield electron emission from a cathode tip, (ii) volume and surface electron current distribution, and (iii) main interaction phenomena between energetic electrons and the MP. Then, the results are exposed and discussed in the fourth section. The last section summarizes the main steps and conclusions of this work.

\section{PHENOMENOLOGICAL MODEL}

The cathode considered in the model is metallic and assumed to have an irregular (rough) surface. Further, it is assumed that the macroscopic current recorded between the cathode and the anode is essentially due to one major tip on the surface, as long as the breakdown does not occur. This is often called "dark current" and it should be kept as low as possible for safe operation at high voltage under vacuum [16]. In this model, a smooth protrusion is considered but, actually, nanoprotrusions can exist on the protrusion and multiply the field enhancement following the Schottky's conjecture $[34,35]$. However these nanoprotusions are too small to affect the emitted current in our model.

The distance between the major tip and the surrounding neighbor tips at the cathode surface is considered large enough such that the collective effects (thermal or electrostatic) could be neglected. The electrons emitted from the tip are accelerated in the interelectrode gap.

The most often mentioned hypothesis in the literature considers that MP comes from the anode [36,37]. Indeed, Eastham and Chatterton [25] experimentally show that $90 \%$ of the MPs originate from the anode. These MPs are due to "hot spots" created at the anode by the electrons emitted from the cathode. This type of hot spot is taken responsible for the release of a positively charged MP when the yield stress of the metal at the hot spot becomes lower than the surface stress of the applied electric field [38]. The clump thus detaches and starts to move in the interelectrode gap under the electric field effect. Other phenomena can explain why MPs leave the anode, for instance, a different surface treatment (or material) between the cathode and the anode. On the contrary, if the MPs are released by the cathode tip, then the tip flattens and the current drops down. However, if 

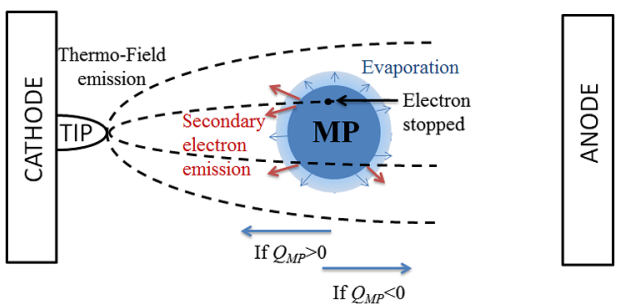

FIG. 1. Sketch showing the phenomena occurring in the interelectrode gap. Both the tip (on the cathode) and the microparticle (MP) are at micrometer $(\mu \mathrm{m})$ scale, while the anodecathode gap is in the $\mathrm{mm}$ range (the figure is not to scale). The dashed lines denote the trajectories of thermofield emitted electrons from the tip due to the high anode-cathode voltage.

the thermofield electron emission from the tip survives after the MP leaves the electrode, the electron bombardment induces high secondary electron emission from the MPs surface that can reverse its charge and bring it back to the cathode.

During its flight, the MP interacts with the electrons released by the tip. This bombardment of the MP by the high energy primary electron has several consequences (see Fig. 1): (i) The primary electrons emitted from the tip lose energy (stopping power) when they impact the clump and heat it up, consequently the MP radius decreases due to evaporation and sometimes the complete vaporization occurs. (ii) The MP charge can be modified by two different processes: (a) Electrons can be stopped inside the clump (MP). This negative "charge collection" reduces its initial positive charge. Hence the total charge decreases and can even reverse to a negative value. (b) The interaction between the primary energetic electrons and the clump (MP) can induce secondary electron emission. The total charge changes according to the number of electrons released with respect to the impinging energetic electron.

This charge variation modifies the electric force acting on the MP and consequently its energy, flight time, and trajectory. Indeed, if the MP charge becomes negative, the clump can even stop and start to fly back to the anode or if many of the thermofield primary emitted electrons are intercepted by the microparticle, it can heat up and vaporize in the interelectrodes gap.

\section{MODEL BACKGROUND}

\section{A. Electric field distribution in the interelectrode gap}

The simulated geometry is represented in Fig. 2. The electric field distribution in the interelectrode gap can be divided in three zones. Near the tip (different sizes have been treated in this article: the largest treated here is $H_{\text {tip }}=$ $10 \mu \mathrm{m}$ height and $R_{\text {tip }}=0.97 \mu \mathrm{m}$ radius at its base on the cathode, while the smallest one is $H_{\text {tip }}=0.2 \mu \mathrm{m}$ and $R_{\text {tip }}=0.003 \mu \mathrm{m}$ ), the electric field is enhanced by the sharp shape of the apex. This region is limited to the

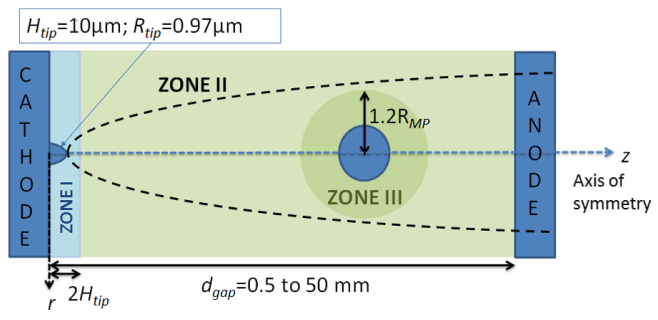

FIG. 2. Representation of the three characteristic zones of a specific electric field, as used in this model. Zone I: field enhancement close to the emissive tip extending on twice the tip height (on the figure is indicated the larger value of this zone for a tip of $10 \mu \mathrm{m}$ height and $0.97 \mu \mathrm{m}$ radius at its base); zone II: zone of uniform field (gap lying from 0.5 to $5 \mathrm{~mm}$ ); zone III: spherical field produced by the charge of the MP surrounding it. The figure is not drawn to scale.

vicinity of the cathode (zone I). The enhancement zone is taken equal to $2 H_{\text {tip. }}$. After this zone the field is uniform and given by the macroscopic value equal to

$$
\vec{E}=-\frac{V_{\text {app }}}{d} \vec{e}_{z}
$$

where $V_{\text {app }}$ is the applied voltage at the anode (the cathode is taken grounded). The effect of the space charge is neglected. Further analysis including the space charge will be presented in a future communication.

The electric field distribution in this zone is $2 \mathrm{D}$ axisymmetric. The Laplace equation is solved:

$$
\begin{aligned}
\Delta V & =0 \\
E & =-\vec{\nabla} V .
\end{aligned}
$$

The boundary conditions are

$$
\begin{array}{rlrl}
V_{\text {cathode }} & =0 ; & & \left.E_{z}\right|_{\text {end-zoneI }}=\left.E_{z}\right|_{\text {macro }}=\frac{-V_{\text {app }}}{d} ; \\
\left.E_{r}\right|_{\text {end-zoneI }}=0 ; & \left.E_{r}\right|_{\text {right-border }}=0 .
\end{array}
$$

$E_{r}$ and $E_{z}$ are respectively the radial $(r)$ and the axial (z) components of the electric field.

Zone III denotes an area where the electric field created by the MP (of radius $R_{\mathrm{MP}}$ ) is significant. The radius of this zone is taken equal to $1.2 R_{\mathrm{MP}}$. The field in this zone is considered equal to the superposition of the electric field in the interelectrode gap and the local electric field created by the clump. The local electric field induced by the MP at a location $N$ can be written as

$$
\vec{E}_{\mathrm{MP}}(N)=\frac{Q_{\mathrm{MP}}}{4 \pi \varepsilon_{0}|\overrightarrow{O N}|^{3}} \overrightarrow{O N},
$$

where $O$ is the MP center and $R_{\mathrm{MP}}<|\overrightarrow{O N}|<2 R_{\mathrm{MP}}$. It represents the electrostatic field created by a sphere of 
charge $Q_{\mathrm{MP}}$ uniformly distributed on the MP outer surface. With the superposition of these fields, the axisymmetric geometry is broken and the problem becomes fully 3D. Hence, the electrons passing close to the MP cross a 3D field region, in spite of the axisymmetric situation with respect to the tip. Also, in the general case, the MPs can leave the anode off axis.

This study focuses on the MP leaving the anode, and most of the cases concern MPs traveling along the symmetry axis of the tip, since the electron current distribution on the anode is maximum on this axis (Fig. 5). However, several cases have been analyzed considering off-axis MPs released from the anode, and they are explicitly indicated in the manuscript (see Sec. IV C). First, the processes concerning the microtip and the clump will be detailed.

\section{B. Electron emission}

The electron emission on the tip surface is calculated following the Murphy and Good model (Wentzel Kramers Brillouin approximation) [39]. The tip surface temperature and the applied voltage over the gap $\left(V_{\text {app }}\right)$ are the two input parameters of the present model. The electric field at the surface of the tip is determined by solving the Laplace equation [Eqs. (2) and (3)]. The tip surface temperature is given by the temperature distribution in the whole microprotrusion which is the balance between the Joule heating effect, the temperature at the base of the cathode (assumed huge compared to the tip and acting as a thermostat at $300 \mathrm{~K})$ and the Nottingham effect [10] at the tip apex surface. The Nottingham effect has an important role in the tip heating $[40,41]$. Note that the losses by radiation are negligible. The Joule heating is induced by the electron current flowing along the tip. The current conservation is fulfilled in the tip volume, leading to the electron emission at the tip surface, but mainly at its apex for two reasons. First, the apex is the region of the highest temperature and, second, it is the region with the highest electric field, due to the highest curvature, so field enhancement.

The problem is reduced to a $2 \mathrm{D}$ axisymmetric timedependent model, assuming a perfect azimuthal symmetry of the tip. All the derivatives of the physical quantities with respect to the azimuthal coordinate are taken to be zero. The emitter is set on a cylinder whose size is much bigger than the tip, namely the cathode, acting as a thermal reservoir (Fig. 3).

A complete description of the model and the thermofield model equations with their boundary conditions can be found in [42]. The coupled physics is solved by combining a time dependent finite elements solver and a nonlinear solver (Newton-Raphson), part of the Comsol Multiphysics ${ }^{\mathrm{TM}}$ library [43].

Figure 4 shows different results obtained with the electron emission model for a big titanium tip. The dimensions of the elliptical microtip are $H_{\text {tip }}=10 \mu \mathrm{m}$

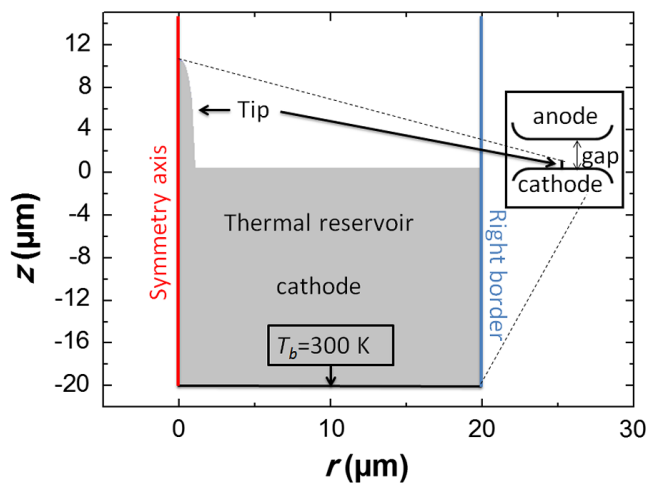

FIG. 3. Magnification of the vicinity of the tip taken with an elliptic shape. The tip is represented with its thermal reservoir (thermostat) which is the cathode. The inset shows the interelectrode gap (the size of the tip is not to scale).

and $R_{\text {tip }}=0.97 \mu \mathrm{m}$ (shown at the left side of Fig. 4). The 2D distribution of the $z$ component of the electric field is represented in Fig. 4(a) only for the apex of the tip where it is enhanced, typically for a radius of $100 \mathrm{~nm}$, which represents about $10 \%$ of the base radius of the tip.

The 2D distribution of the current density is shown in Fig. 4(b) and the emitted current density at the surface around the apex in the inset [Fig. 4(b') the abscissa axis lies on $150 \mathrm{~nm}$ only]. As expected, the current density is the highest at the apex because the field is enhanced at this location. The emission radius is considered equal to $150 \mathrm{~nm}$ for the biggest tip considered in this study, since the current density decays by more than 3 orders of magnitude beyond this radius.

The 2D temperature distribution is shown in Fig. 4(c): the temperature is higher at the apex due to the Joule effect.

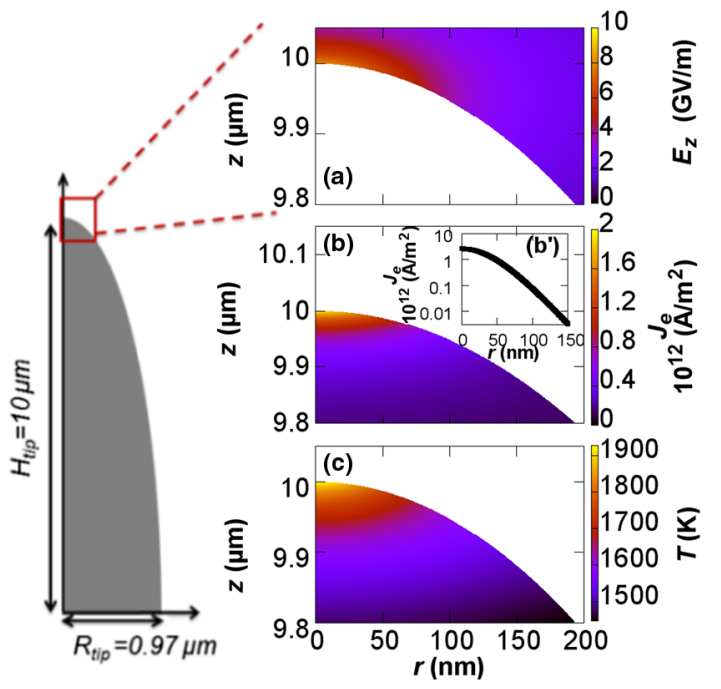

FIG. 4. 2D distributions of the electric field (a), current density (b), and temperature (c) calculated for an imposed uniform macroscopic field $\mathrm{E}_{\text {macro }}=5 \times 10^{7} \mathrm{~V} / \mathrm{m}$ and the big tip. Surface normal current density is represented in $\left(b^{\prime}\right)$, the inset of panel (b). 
It should be noted that in this case the Nottingham effect provides a heating flux.

Similar calculations have been performed for smaller tips (not shown in detail, but their effect is discussed in this article). The smallest one considered here is $200 \mathrm{~nm}$ height (the enhancement field $\beta$ is equal to 50), corresponding to a very well-polished cathode surface. The radius of the emission area, in this case, is reduced to $3 \mathrm{~nm}$ only. Below this size, the tip has an emission area too small and the use of Murphy-Good's model is not consistent anymore, because in the latter emission model, the potential barrier is determined from a plane surface and the curvature effect is neglected. For a nanometer tip, the curvature effect cannot be neglected. Forbes [44] and Fisher [45] proposed a corrective term in the potential barrier to describe this effect and Kyrtisakis et al. [46] use a 3D Wentzel-Kramers-Brillouin approximation to show a decrease of the effective emission area when the ellipsoid becomes more elongated for a constant field.

Therefore, the present model addresses a large variety of tips, from very small of $200 \mathrm{~nm}$ to large tips of $10 \mu \mathrm{m}$ height, covering the realistic roughness obtained after regular machining and polishing of the metal cathodes.

\section{Current distribution and surface distribution}

The previously calculated emission electron current is used as a source term for the electric charging of the MP traveling in the interelectrode gap. In this study tip emitted electrons are called primary electrons because they act directly on the MP, inducing several phenomena such as electric charge modification, evaporation, etc. For the present model, the electron emission is considered to follow the emission current density distribution represented in Fig. 4, independent of the applied field. In other words, only the amount of electrons is changed (current), but not the space distribution of primary electrons.

This current density is used as input data of a 3D model that follows the trajectories of primary electrons in the interelectrode gap. The initial radial coordinate $\left(r_{\text {init }}\right)$ of each primary electron is taken in the vicinity of the topmost area of the tip. The value of $r_{\text {init }}$ is randomly chosen, but weighted according to the current density distribution represented in Fig. 4(b'), using a Monte Carlo (MC) method. Knowing $r_{\text {init }}$, the $z$-coordinate $\left(z_{\text {init }}\right)$ is determined from the equation of the ellipse (the assumed shape of the tip):

$$
z_{\text {init }}=H_{\text {tip }} \sqrt{1-\frac{r_{\text {init }}^{2}}{R_{\text {tip }}^{2}}} .
$$

For convenience, the 3D model operates in Cartesian coordinates $(x, y, z)$. Hence, the radial coordinate $r_{\text {init }}$ is assigned to $x_{\text {init }}$ and $y_{\text {init }}$ both determined by assuming the uniform azimuthal emission probability. The initial velocity of the electrons is neglected because the emission energy is much smaller (typically $1 \mathrm{eV}$ ) [39] compared to the velocity (kinetic energy) reached in the vicinity of the tip and further in the interelectrode gap. It should be noted that the field is enhanced and electrons are freely accelerated under vacuum (no collisions with any background gas). The electrons quickly reach relativistic velocities due to the high electric field close to the tip and they continue to be accelerated in the electrode gap. Electron trajectories are calculated by a Monte Carlo method using the 3D field calculated as explained Sec. III A.

To respect the stability criterion of this MC model, the chosen time step must respect the Courant-Friedrichs-Lewy (CFL) condition. It requires a very low time step in zone I because the potential (electric field) decreases sharply. Therefore, the chosen time step is $1.0 \times 10^{-14} \mathrm{~s}$.

Figure 5 shows the normalized electron density when they reach the surface of the anode, for an interelectrode gap $d=$ $500 \mu \mathrm{m}$ and $V_{\text {app }}=25 \mathrm{kV}$, gap voltage. The symmetry axis corresponds to $x=0 \mathrm{~mm}$ and $y=0 \mathrm{~mm}$. The primary electron beam limit intercepting the anode is represented by the dotted line [Fig. 5(a)]. The electron beam expands under the effect of the electric field in zone I. In Fig. 4(b'), the total emission area is given by a small dome of only $150 \mathrm{~nm}$ radius at the top of the tip, while the beam interception radius reaches $90 \mu \mathrm{m}$ at the anode. This normalized electron density is represented as a function of the radius in Fig. 5(b) for the highest tip $\left(H_{\text {tip }}=10 \mu \mathrm{m}\right)$. Notice that both, the initial and final primary electron distribution, presents a similar shape to the radial position, even if the slope is larger at the tip. The area covered by the primary beam changes along $z$, according to the electric field given by Eq. (3).
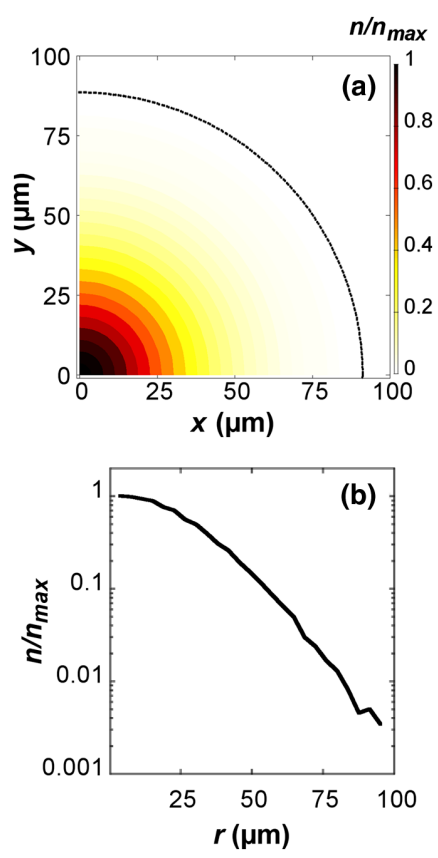

FIG. 5. (a) 2D polar distribution of the normalized surface number density of electrons impinging the anode (color scale of top panel) and (b) the same distribution expressed versus radial position. 
For the smallest tip $\left(H_{\text {tip }}=200 \mathrm{~nm}\right)$, as said above (Sec. III B), the emission apex radius is only $3 \mathrm{~nm}$ and the beam interception radius reaches $14 \mu \mathrm{m}$ at the anode, for a $500 \mu \mathrm{m}$ gap (not shown). Even for small tips, it completely covers the trajectories for MPs $<10 \mu \mathrm{m}$, summarized in Fig. 12(a). Obviously, for the case of larger gaps [50 mm, Fig. 12(b)] the primary electron interception radius on the anode exceeds $100 \mu \mathrm{m}$ radius, the largest MP discussed in Sec. IV B.

\section{Microparticle model}

The microparticle is assumed to be spherical and metallic (good electrical conductivity) being initially in contact with the anode. The MP detaches from the anode surface if the Coulomb forces overcome the elasticity force [4]. Hence the MP starts to move in the interelectrode space, under vacuum, towards the cathode, driven by the macroscopic electric field. Its initial charge $Q_{\mathrm{MP}}$ [47] is controlled by the electrode surface electric field. This charge is expressed as follows:

$$
Q_{\mathrm{MP}}=6.58 \pi \frac{V_{\mathrm{app}}}{d} R_{\mathrm{MP}}^{2} .
$$

The factor 6.58 is the enhancement coefficient of the electric field created by the MP itself at the anode, after [48].

\section{MP-electron interaction cross section}

It is assumed that the MPs, if several are released simultaneously at the anode, are isolated from each other and do not mutually interact as in the orbit-limited motion (OLM) approach [49]. The difference with the OLM theory is the superposition of the electric field in the interelectrode field over the MP field, leading to the 3D field around the MP. Electrons are launched from the tip as described in Sec. III B (zone I). When one electron approaches the MP, its trajectory is influenced by the MP electric field and it is deflected according to the local electrostatic force. In order to reduce the computation time, the impact parameter $(\rho)$ giving the maximum cross section $\left(\sigma_{\mathrm{MAX}}=\pi \rho^{2}\right)$ was attempted to be found. $\rho$ was estimated such as MPelectron interaction has a significant effect on the primary electron trajectory. This impact parameter has been calculated for different electron initial velocities. As an example, the results obtained for a MP $\left(R_{\mathrm{MP}}=1 \mu \mathrm{m}\right.$ and charge $Q_{\mathrm{MP}}=9 \times 10^{-15} \mathrm{C}$ ) have been represented in Fig. 6 .

The maximum impact parameter is only $20 \%$ larger than the MP radius, namely $\rho=1.2 R_{\mathrm{MP}}$ for all MPs studied here, with the radius varying from 0.1 to $100 \mu \mathrm{m}$, interacting with a primary electron of $10^{5} \mathrm{~m} / \mathrm{s}$ represented by the blue line with circles in Fig. 6. This impact parameter decreases for higher primary electron velocities $\left(10^{7} \mathrm{~m} / \mathrm{s}\right.$, represented with the black curve, Fig. 6) because the effect of the electric field created by the clump is less efficient.

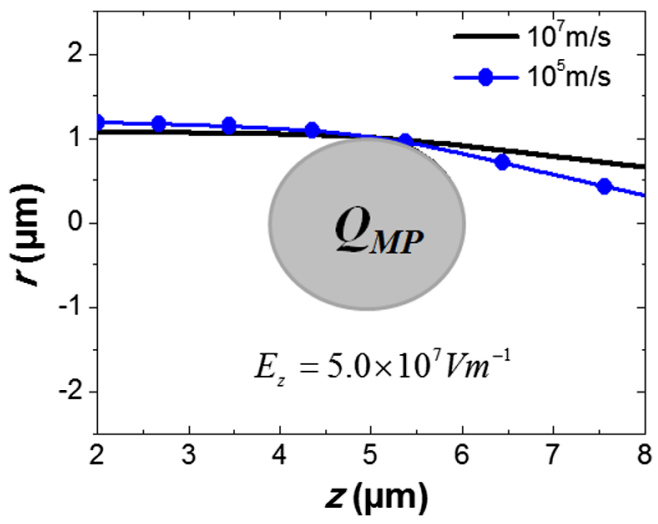

FIG. 6. Primary electron trajectories passing close to the MP for two velocities. The deflection occurs only very close of the MP.

Note that the velocity of almost all the primary electrons in the interelectrode gap exceeds $10^{7} \mathrm{~m} / \mathrm{s}$. Practically, the interaction cross section approaches the section of the MP. This parameter $(\rho)$ defines the interaction zone III described in Sec. III A (Fig. 2).

\section{Secondary electron emission}

Different phenomena may occur at the surface of the clump caused by the impact of primary electrons. The electrons penetrate into the MP and lose part of their energy on the way. This loss is called the stopping power and it depends on the electron energy just before the interaction. The ESTAR database [50] was used to determine the stopping power based on the Bethe theory [51,52] and experimental data [53]. This energy loss by the primary electrons heats up the MP. In the model presented here, the clump is assumed to be leaving the anode at the vaporization temperature. Hence, this energy fraction recovered from the stopping power is added to the latent heat of the material leading to its partial evaporation. If the accumulated energy brought by the primary electrons is high enough, the MP can be completely vaporized (discussion in Sec. IVA).

The MP charge is distributed at the surface, since it is assumed metallic. However, the net charge can change in two ways: (i) an electron can lose all of its kinetic energy inside the clump being stopped and absorbed or (ii) one or several secondary electrons can be emitted by the MP irradiated by the energetic primary electron, changing its charge. The situation is well known in the case of a semiinfinite plane [54]. In the case of dusty plasmas, the electrons being of relatively low energy, it may be considered that the MPs act as semi-infinite planes [55]. In the present case, due to the high energy of the primary electrons being accelerated in vacuum under high electric fields, they can traverse the entire MP. The approach to describe the secondary emission in this case has been developed by Chow et al. [56]. They assume that the production of secondary electrons is proportional to the 
stopping power of the primary electron and it exponentially decays with path length $(l)$ inside the MP. The secondary current $i_{s}$ due to the primary current $i_{p}$ that has penetrated the MP in the space range $[x, x+d x]$ can be written as

$$
\mathrm{d} i_{s}=-K i_{p} \frac{\mathrm{d} E_{p}}{\mathrm{~d} x} e^{-\alpha l(r, x, \theta, \varphi)} \mathrm{d} x
$$

where $K$ is the inverse of the energy required to produce a secondary electron, $\frac{\mathrm{d} E_{p}}{\mathrm{~d} x}$ the stopping power, $\alpha$ the inverse of the absorption length of the electrons, and $l(r, x, \varphi, \theta)$ the distance that a secondary electron must travel to reach the MP surface, from the creation location.

It can be shown that the distance $l(r, x, \theta, \varphi)$ is given by

$$
\begin{aligned}
l(r, x, \theta, \varphi)= & -\left[r \sin (\theta) \cos (\varphi)+\cos (\theta)\left(\sqrt{R_{M P}^{2}-r^{2}}-x\right)\right] \\
& +\sqrt{\left[r \sin (\theta) \cos (\varphi)+\cos (\theta)\left(\sqrt{R_{M P}^{2}-R^{2}}-x\right)\right]^{2}-x\left(x-2 \sqrt{R_{M P}^{2}-r^{2}}\right)} .
\end{aligned}
$$

For a spherical MP (Fig. 7), where the notations follow a cylindrical coordinates system with $r$ the radius from the MP center, $x$ is the linear path of one primary electron from the entry position to the location producing a secondary electron (marked with a star on Fig. 7). The angles, $\theta$ and $\phi$ give the direction of the secondary electron with respect to the symmetry axis and the one orthogonal to it, respectively.

By integration, the secondary yield (number of secondary electrons for one primary electron) (denoted $\delta_{s}$ ) can be determined by

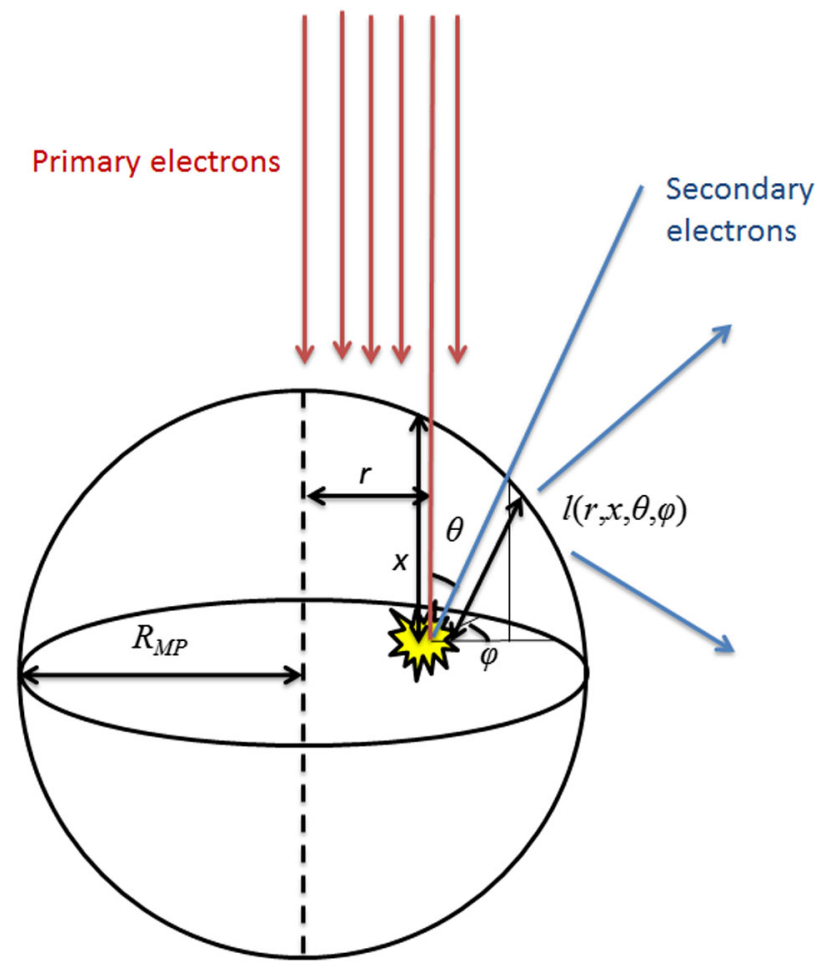

FIG. 7. Secondary electron emission induced by primary energetic electrons for a spherical MP. The location where a secondary electron is created is marked by a star.

$$
\begin{aligned}
\delta_{s}= & \frac{K}{4 \pi R_{M P}^{2}} \int_{0}^{R_{\mathrm{MP}}} r d r \int_{0}^{\min \left(x_{\max }, x_{\text {stopped }}\right)} \frac{d E_{p}}{d x} d x \\
& \times \int_{0}^{\pi} \sin (\theta) d \theta e^{-\alpha l(r, x, \theta, \varphi)} d \varphi,
\end{aligned}
$$

where

$$
x_{\max }=2 \sqrt{R_{M P}^{2}-r^{2}}
$$

and $x_{\text {stopped }}$ is the distance before the primary electron is stopped. As a typical example, Fig. 8 shows the secondary electron yield for a MP of $10 \mu \mathrm{m}$ radius obtained from Eq. (10).

The secondary emission is higher for low energy primary electrons because the fraction of energy lost when they enter the MP is larger and the secondary electrons are created close to the surface. As the primary electron energy increases, the secondary emission decreases because the secondary electrons are created much deeper inside the MP and the primary electrons are stopped inside the particle. Even if they are created in the MP core, these secondary electrons can hardly reach the surface in this case. The minimum of the secondary emission yield if found for

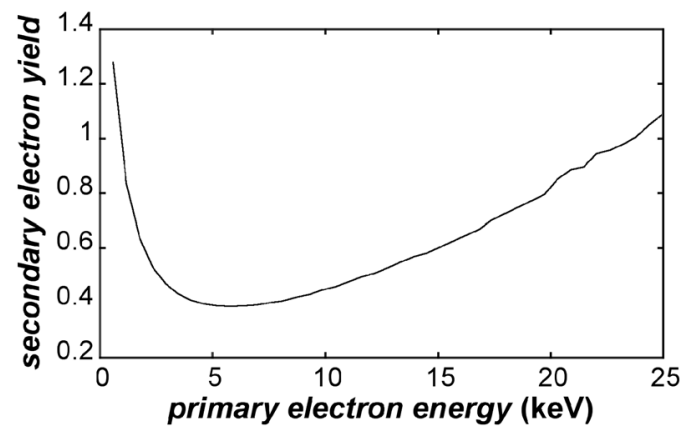

FIG. 8. Secondary electron yield induced by electron bombardment for a $10 \mu \mathrm{m}$ radius MP. 
about $5 \mathrm{keV}$ for a MP of $10 \mu$ m radius (Fig. 8). For very high energies, the production of secondary electrons increases again since the primary electrons have enough energy to cross through the entire clump and then can produce more secondary electrons close to the surface, when they enter and they exit the MP. Newly created secondary electrons must have a sufficient energy to be able to escape the microparticle attractive potential if it is positively charged. The secondary electrons exit mostly perpendicular to the surface with an average energy of $10 \mathrm{eV}$ [54]. This emission area $A_{e e}$ is defined as the surface on the MP from where an electron can escape the MP recapture electric field. The escaping coefficient is defined as the ratio of the emission surface $A_{e e}$ on the total MP surface $A_{\mathrm{MP}}$ and it is shown in Fig. 9 for several sizes of MPs.

When the MP charge is low, the effect of the electric field surrounding the MP is not strong enough to stop the secondary electrons and they can escape (escaping coefficient $\sim$, Fig. 9). When the MP charge increases, the emission rate decreases and beyond a certain MP charge the secondary electrons cannot escape anymore. This limit varies with the size (or charge) of the MP. If two clumps have the same charge but different sizes, the electric field created at the surface will be larger around the smallest one [see Eq. (7)] and it will be more difficult for a new electron to escape.

\section{Thermofield emission of the MP}

For high temperature and for a high electric field, the titanium MP (with a work function $\varphi=4.5 \mathrm{eV}$ [57]) can emit electrons by thermofield emission. In this study, for $V_{\text {app }}=25 \mathrm{kV}$ and $\mathrm{d}=0.5 \mathrm{~mm}$, and MP radii lower than $10 \mu \mathrm{m}$, this current is always lower than $1 \mu \mathrm{A}$. Therefore, the thermofield current of the MP is neglected in our model.

To sum up, two models have been developed presented as a flowchart in Fig. 10. In the first model, called "electron model," characterized by the initial tip geometry, the field distribution inside the interelectrode gap is determined and the thermofield emission is obtained. The space distribution of the electron emission can be precisely determined as well as the trajectories of electrons reaching the anode. The results of this electron model are used as input for the second model that deals with the "MP dynamics." This

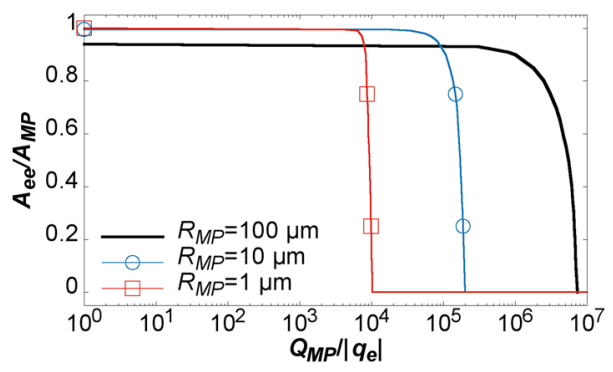

FIG. 9. The escaping coefficient of a secondary electron versus the MP charge number for MPs of different sizes.

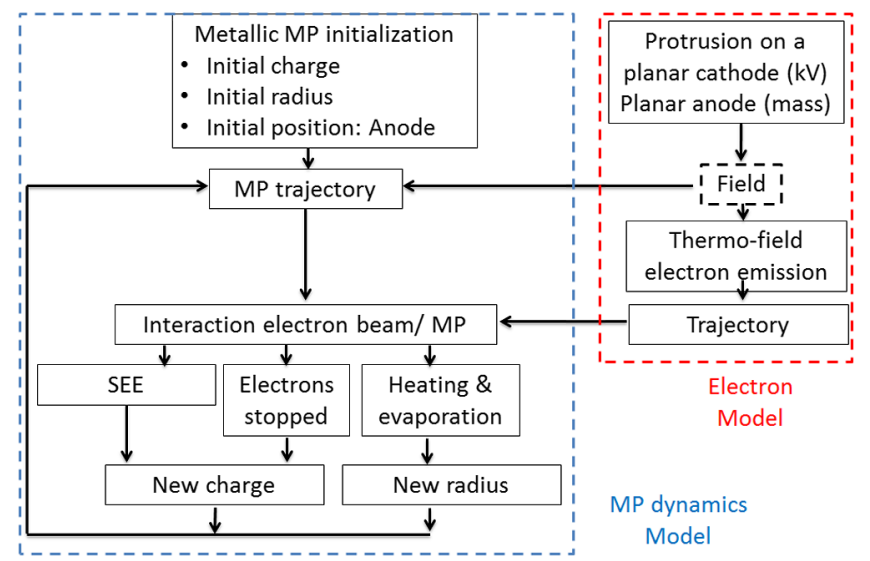

FIG. 10. Model flowchart of the 3D MP dynamics. Two models are connected, one corresponds to thermofield electron emission from an apex (red) and the second to the MP trajectory (blue). SEE denotes the secondary electron emission.

second model treats the interactions between the MP and the electron beam. Several effects on the MP are identified, due to these interactions: heating and evaporation of the MP caused by the power loss of the energetic electrons striking the MP and charge variation at the surface of the MP due to the capture of the beam electron or, on the contrary, to secondary electron emission induced by energetic electrons. These phenomena affect the electrostatic force acting on the MP and consequently its dynamics.

\section{RESULTS AND DISCUSSION}

\section{A. Trajectory for a test MP}

The transit of one MP of $10 \mu \mathrm{m}$ initial radius when it leaves the anode is represented as a test clump evolving in the interelectrode space, along the emissive tip axis. The interelectrode gap $(d)$ and the voltage gap $\left(V_{\text {app }}\right)$ are respectively equal to $0.5 \mathrm{~mm}$ and $25 \mathrm{kV}$. The emitted current at the surface of the tip is set as an input parameter.

For very low current (here $1 \mu \mathrm{A}$ ), the MP crosses the gap [Fig. 11(A.a)] and reaches the cathode in $\sim 20 \mu$ s. The charge decreases and becomes negative when it approaches the cathode [ 100 $\mu$ m; Fig. 11(A.b)]. A substantial number of primary electrons are collected by the MP because the beam diameter decreases near the cathode. In spite of the charges collected, the current density is not high enough to efficiently heat up the MP. Consequently, the evaporation is absent and the MP radius stays unchanged during the flight between the two electrodes [Fig. 11(A.c)]. This behavior is called "One way" in Fig. 11.

For a current of $10 \mu \mathrm{A}$ emitted by the tip, when the MP leaves the anode, it traps enough electrons to reduce their charge and consequently their velocity. The charge becomes negative after $\sim 20 \mu$ s [Fig. 11(B.b)], further from the cathode compared to the previous case. Reversing its charge the MP slows down and it can even turn back to the 


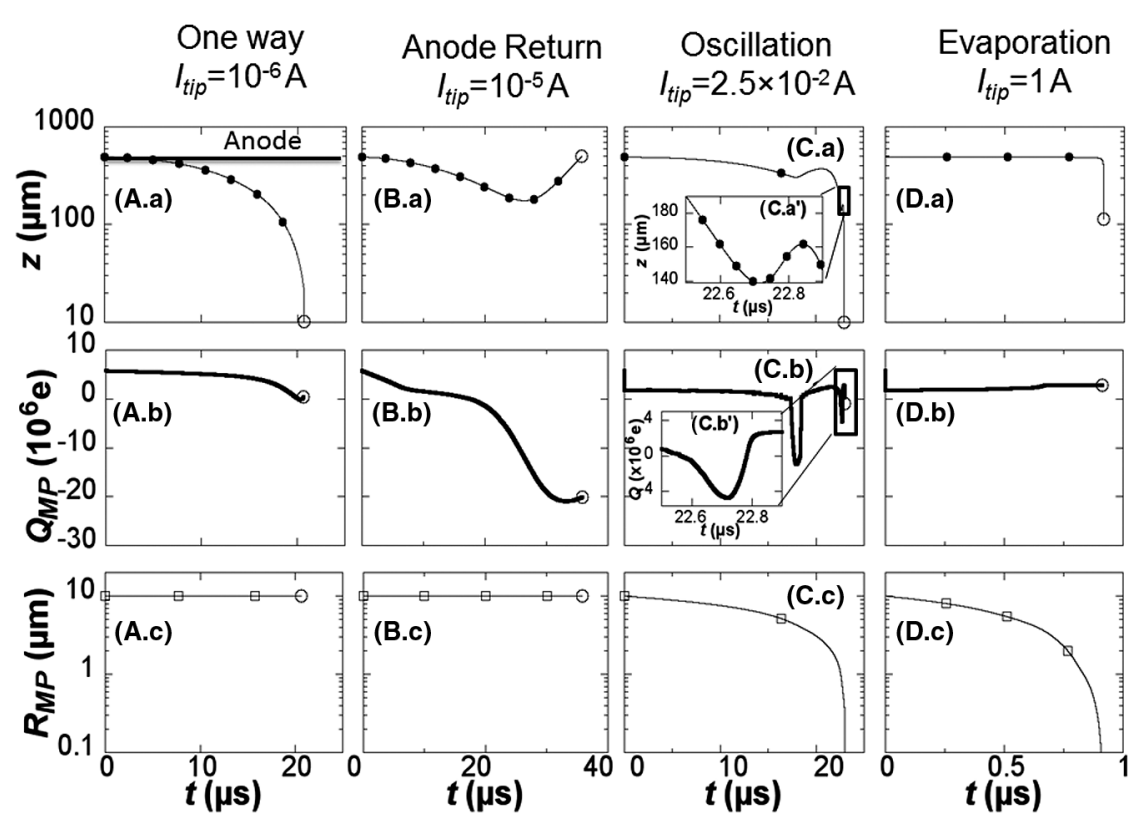

FIG. 11. Dynamics regimes of a test MP for different emission currents. The rows represent the time evolution of: (a) the flight distance $(\mathrm{z})$ from anode $(500 \mu \mathrm{m})$ towards the cathode $(\mathrm{z}=0)$; (b) the charge $\left(\times 10^{6} \mathrm{e}\right)$ of the MP; and (c) the MP radius $\left(R_{\mathrm{MP}}\right)$. By columns, the first corresponds to "one way" trajectory from the anode to the cathode and low tip current. The second column corresponds to a MP back-attracted to the anode. The third column corresponds to an oscillating MP before reaching the tip. The fourth column represents an MP finishing fully evaporated due to the very high primary current. For each current, $\mathrm{V}_{\text {app }}=25 \mathrm{kV}$ and $\mathrm{d}=0.5 \mathrm{~mm}$ have been kept fixed as well as the initial particle radius. Animations for each regime are available on-line [58].

anode (“Anode Return”). The MP size stays constant [Fig. 11(B.c)], as in the previous case.

For a significant primary current of $25 \mathrm{~mA}$, the MP quickly collects enough energetic electrons to balance its initial positive charge so it becomes neutral during the first microseconds [Fig. 11(C.b)] then the charge stays negative and slightly increases in absolute value over the next $15 \mu \mathrm{s}$. Even if the large amount of primary electrons irradiating the MP is effectively balanced by the secondary electrons, preserving thus the total absolute charge close to zero (slow MP), the heating effect induced by the bombardment of the primary electrons on the MP is no longer negligible. Hence, the size of the MP decreases significantly [Fig. 11(C.c)]. Decreasing its size and approaching the cathode (primary electron energy decreases), the secondary emission yield decreases too [Eq. (10) and Fig. 8 around the minimum]. Thus, the MP suddenly starts accumulating electrons (at $\sim 15 \mu \mathrm{s}$ ). The charge reverses and the MP moves back towards the anode at $z=300 \mu \mathrm{m}$ [Fig. 11(C.a)]. However, its size continuously decreases [Fig. 11(C.c)], and the primary electrons can cross the MP easier, increasing the secondary electron yield (crossing twice the MP surface) and leading to a second reversal of the charge, back to a positive state. The electric field created by the MP (zone III) slightly slows down the primary electrons but the decrease of their kinetic energy is too low to significantly affect the secondary electron emission. For example, in Fig. 12, the

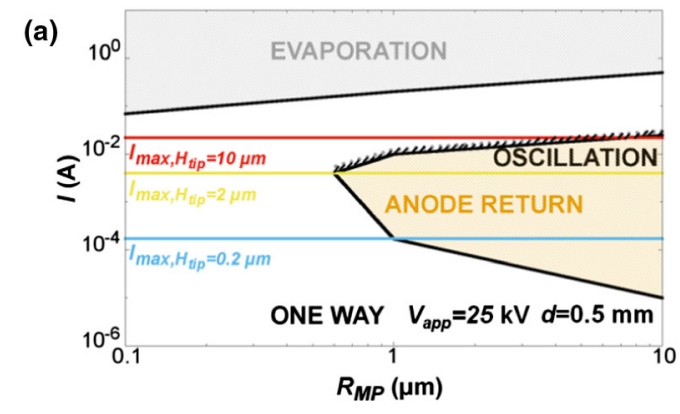

(b)

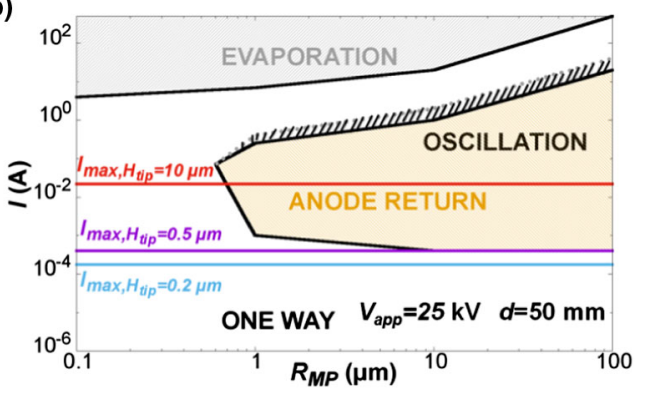

FIG. 12. Different regimes of MPs dynamics versus their sizes. The figure corresponds to a constant voltage $\mathrm{V}_{\text {app }}=25 \mathrm{kV}$ and two gap distances (a) $\mathrm{d}=0.5 \mathrm{~mm}$ and (b) $\mathrm{d}=50 \mathrm{~mm}$. The horizontal lines corresponds to the maximum emitted current by three tips, the largest one $\left(H_{\text {tip }}=10 \mu \mathrm{m}\right.$, red), the average size $\left(\mathrm{H}_{\text {tip }}=2 \mu \mathrm{m}\right.$, green and $0.5 \mu \mathrm{m}$, blue $)$ and the smallest one $\left(H_{\text {tip }}=0.2 \mu \mathrm{m}\right.$, cyan). 
MP did its first oscillation at $0.3 \mathrm{~mm}$, and primary electrons have already $15 \mathrm{keV}$. Due to the Coulombian repelling forces, the electron loses $\sim 1 \mathrm{keV}$. Figure 8 shows that the secondary electron emission does not change significantly between 14 and $15 \mathrm{keV}$. Hence, the MP approaches once again the cathode.

The same scenario (second oscillation) occurs again at $z=160 \mu \mathrm{m}$ [inset Fig. 11(C.a')]. Hereafter, it does not oscillate again because the size of the MP becomes too small $[<1 \mu \mathrm{m}$, Fig. 11(C.c)] and its charge is governed by the secondary emission, i.e., it stays positive. As the MP mass becomes lower and lower, the flight time towards the cathode is too short for the MP to exhibit another oscillation. The clump reaches the cathode as a nanoparticle and not as a microparticle. Let us note that Latham $[4,59]$ assumes that the oscillation behavior is caused by an MP bouncing on the electrodes whereas the present results show that the MP can also oscillate due to the charge variation caused by the charge balance on its surface due to primary electron collection and secondary electron production.

If the MP is exposed to a very high electron current $(0.1 \mathrm{~A})$, the MP evaporates very fast and its size decreases. Beyond this limit $(0.1 \mathrm{~A})$, the MP never reaches the electrodes and it evaporates in the interelectrode gap [Figs. 11(D.a)-(D.c)].

\section{B. Scenarios of the MP dynamics}

The four scenarios found in the previous section have been identified for one MP of $10 \mu \mathrm{m}$ initial radius. They correspond to the four columns in Fig. 11 labeled "One Way" for the MP moving straightforward from the anode to the cathode; "Anode Return" for the MP reversing its charge, and consequently the direction of the movement resulting in the trajectory ending on the same electrode that it left from; "Oscillation" for the MP reversing the charge many times in the gap between the electrodes, but finishing often as nanoparticles (due to intense evaporation) on the cathode; and "Evaporation" for the MPs that are transformed in vapors due to the intensive heating by the primary electron current. Obviously, the latter ones never reach the electrodes.

Based on these scenarios, it is possible to extend the study to a family of MPs of several radii, lying between $100 \mathrm{~nm}$ to $100 \mu \mathrm{m}$. Also, two distances between the electrodes (anode and cathode) have been analyzed, for a constant voltage drop $\left(V_{\text {app }}=25 \mathrm{kV}\right)$. The small gap $(d=0.5 \mu \mathrm{m})$ corresponds to the typical distance between the acceleration grids of the accelerators, while the large gap $(d=50 \mathrm{~mm})$ is typical for the bushing design [2].

Figure 12(a) shows that small MPs $\left(R_{\mathrm{MP}}<0.6 \mu \mathrm{m}\right)$ will fly straight to the cathode and start to vaporize in the case of small interelectrode gap $(0.5 \mathrm{~mm})$ under $25 \mathrm{kV}$. But, it cannot completely evaporate during its transit, regardless of the size of the tip $\left(0.2 \mu \mathrm{m}<H_{\text {tip }}<10 \mu \mathrm{m}\right)$. The complete vaporization of the MP is not possible because the maximum current that even a large tip can emit is still lower than the threshold of electron current required to vaporize the MP, namely $90 \mathrm{~mA}$ and for the smallest MP considered here of $100 \mathrm{~nm}$ diameter. This maximum current represents the amount of electrons which leads to the emissive tip vaporization by Joule effect. When the tip vaporizes, it flattens and the electron emission decreases. The maximum emitted current is respectively equal to 0.17 and $22 \mathrm{~mA}$ for two extreme elliptical titanium tips of $H_{\text {tip }}=0.2 \mu \mathrm{m}$ (cyan) and $H_{\text {tip }}=10 \mu \mathrm{m}$ (red).

For larger MPs $\left(R_{\mathrm{MP}} \geq 0.6 \mu \mathrm{m}\right)$, new regimes appear. If the MP captures more electrons, then it returns back to the anode due to its charge reversing for low currents $(0.3 \mathrm{~mA}$ for $R_{\mathrm{MP}}=1 \mu \mathrm{m}$ and one decade less for $R_{\mathrm{MP}}=10 \mu \mathrm{m}$ ), regardless of the size of the tip. For the tips taller than $2 \mu \mathrm{m}$, the emitted electron current is high enough to reduce the size of MP during its flight due to its partial evaporation leading again to a direct flight (previous described regime). Hence, the MPs reach the cathode. An unstable regime exists during this transition, oscillations of the MP are observed in the interelectrode gap (for instance, one MP of $1 \mu \mathrm{m}$ diameter oscillates for a current of $12.5 \mathrm{~mA}$ and a gap of $0.5 \mathrm{~mm})$. For large MPs $(>2 \mu \mathrm{m})$, their partial evaporation is significant if the tip is very high $\left(H_{\text {tip }}=10 \mu \mathrm{m}\right)$ but it is never completed, the electron current provided by the tip being always below the threshold (the upper bold black line).

For larger interelectrode gaps $(50 \mathrm{~mm})$ and the same voltage of $25 \mathrm{kV}$ [Fig. 12(b)], the same four regimes have been observed, but shifted with respect to Fig. 12(a), since the macroscopic electric field is smaller. The emission intensity should be higher to transit from one regimen to another. Therefore, some regimes observed in Figure 12(a) are no longer observed: For a very small tip of $0.2 \mu \mathrm{m}$ (maximum current is given by the horizontal cyan line), only the regime "one way" exists. If the size of the tip increases to $0.5 \mu \mathrm{m}$, the thermofield current becomes sufficiently high to observe large MP ( $>10 \mu \mathrm{m}$ of radius) returning to the anode. However, even for high tips of $10 \mu \mathrm{m}$, the MP evaporation stays impossible, if it is exposed to the current originating only from one tip. The chance of MP "Oscillation" (hashed area) is slightly larger than for small gaps, but fundamentally, the same phenomena occur, with a higher probability for the MP to reach the cathode. As the evaporation case, the oscillation is not possible if only one emitting tip provides the electron current. Let us notice that the oscillation of the MPs has been observed experimentally in the storage rings for $\mathrm{GeV}$ electron beams [60].

The generality of the model presented here will now be discussed. The physical phenomena considered in zone I have been limited to thermofield emission under a constant enhanced field given by the shape of the tip and related to the macroscopic field established in the interelectrode gap. 
In reality, when the MP approaches the tip, the potential barrier decreases, increasing the electron emission of the tip. This emission will negatively charge the MP, but the field is oriented such as it pushes back the clump towards the anode. To understand this phenomenon, the following extreme case is studied. At the beginning of zone I, the MP is taken to have a negative charge equal to the charge that it should have been if it was in contact with the cathode (the opposite case, but the same assumption upon leaving the anode). For this charge, it is easy to find the maximum velocity at the entrance of zone I allowing the MP to be stopped and returning its trajectory due to the enhanced field, i.e., moving away from the cathode (see Fig. 13).

Figure 13 shows that the threshold velocity (black curve) is always lower than the velocity obtained with our model (red curve with squares). Therefore, the inertia gained by the MP in zone II appears to be too high to stop it in zone I before it reaches the cathode. The transition between the "One way" regime (bringing the MPs to the cathode) and the back and forth ("Anode Return" or "Oscillation") is possible only for very negative MPs.

Figure 14 shows that the charge required for the "comeback" situation, i.e., MP trajectory reversal, is larger (absolute values) than the maximum charge that one MP can have if it comes in contact with the cathode $(\times 2$ for $R_{\mathrm{MP}}=1 \mu \mathrm{m}$ and $\times 20$ for $\left.R_{\mathrm{MP}}=10 \mu \mathrm{m}\right)$.

These results show that the additional phenomena that take place when the MP enters zone I have no effect on the dynamics of the MP for very low currents. Latham shows that a MP could bounce when it arrives at the surface of the electrodes $[4,57]$. During the collision between the MP and the electrodes, the MP keeps a part of its kinetic energy (semielastic collision) and takes the charge of the surface. During these multitransit impact phenomena, the kinetic energy increases progressively and becomes significant, enough to induce the vaporization of the MP and the metallic tip of the electrode during a last collision. This phenomenon is possible but it is not described in the present model. In the following, we consider that a MP sticks on the electrode surface after their collision ("crash").

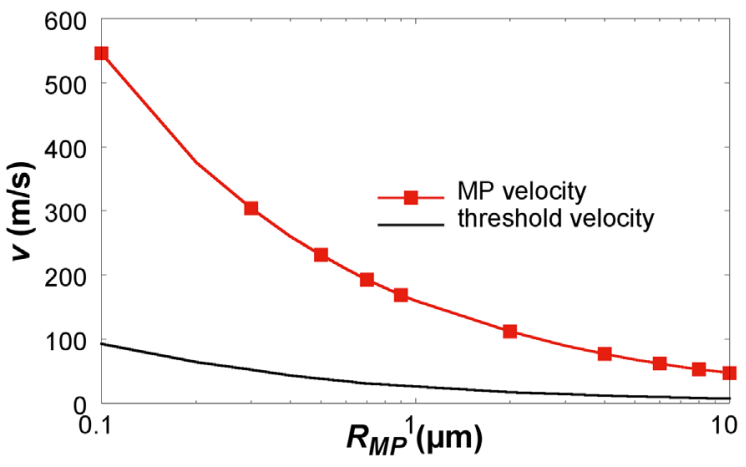

FIG. 13. Comparison of the velocity at the entrance of zone I for $\mathrm{I}_{\text {tip }}=1.0 \times 10^{-7} \mathrm{~A}$ and the threshold velocity obtained for $\mathrm{Q}_{\text {cathode }}$ for different $R_{\mathrm{MP}}$.

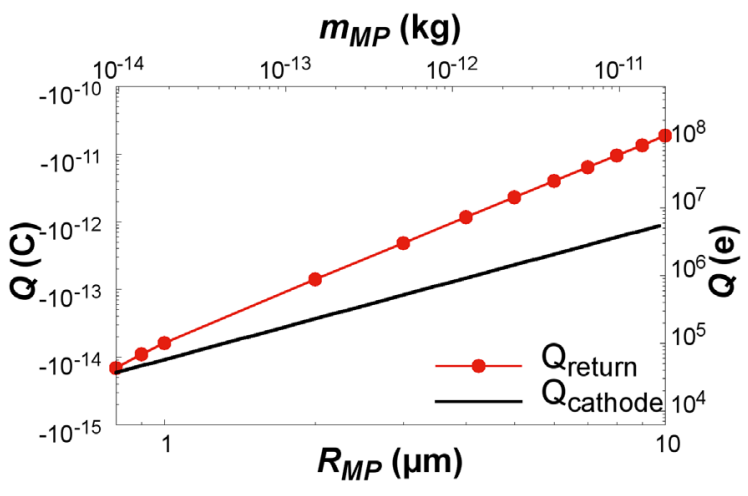

FIG. 14. Comparison of the charge necessary to stop and reverse the MP trajectory $\left(\mathrm{Q}_{\text {return }}\right)$ and the charge of MP in equilibrium with the cathode for different. $R_{\mathrm{MP}}$.

\section{Tip electron emission modifications due to the impinging of MPs on the cathode}

In this section, two extreme cases are analyzed involving the MP's interaction with the emissive tip on the cathode. First, the crash can affect the field emission, and second the thermoionic emission.

\section{Change of field emission when a MP crashes the tip}

Until now, the MP was launched in front of the microtip, along the symmetry axis. In this section, the model is applied to the case where the MP leaves the anode with an offset from the symmetry axis of the tip. The aim is to study the effect of the electric field near the tip, where the radial component of the field is stronger. Hence, one can expect a significant effect on the MP trajectories being eventually deflected towards the apex of the tip.

As found in the previous section, the inertia of the particle is much larger than that of the electron's $(\mathrm{q} / \mathrm{m})$. So, the MP appears to be insensitive to the field variations in the vicinity of the tip apex, since its trajectory stays parallel to the symmetry axis, conserving the initial off-axis shift in its path towards the cathode (not shown). This is interesting as it can now be studied if the MPs are able to crash (or collide) or not with the apex of the tip. If they do, it is important to estimate the effect in terms of electron emission from the tip after the crash.

Figure 15 shows the interaction between the MP and the tip. To characterize the sticking, a new reduced parameter has introduced, defined as $\Delta=\frac{d_{\text {int }}}{R_{M P}}$, where $d_{\text {int }}$ is the distance between the tip axis and the center of the MP.

When the MPs have the radius equal to the tip radius, the maximum value approaches 2 (exactly $\Delta=1.9$ ), approaching the sum of the two radii. The reduced parameter $\Delta$ decays with the MP radius [Fig. 15(b)].

Obviously, when the MP radius has the same size as the height of the roughness, the interaction distance is slightly higher than 1 [blue MP in Fig. 15(a)]. The higher the aspect ratio of the tip, the closer to $1 \Delta$ is. 

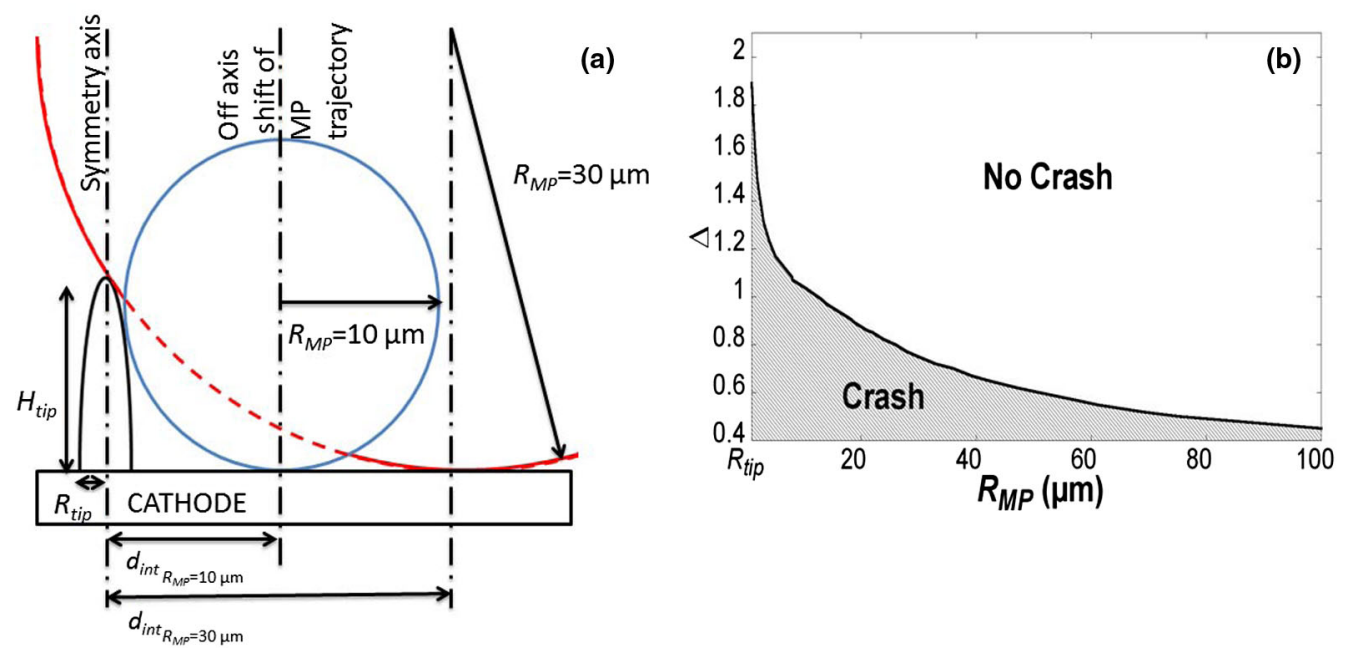

FIG. 15. (a) Sketch of the crash of a large MP touching the tip. (b) Off-axis study. $\Delta$ is a reduced parameter, related to the size of the tip divided by the MP radius $\left(\Delta=\frac{d_{\text {int }}}{R_{M P}}\right)$.

For MPs even larger, for instance, with a radius $R_{\mathrm{MP}} \approx 100 \mu \mathrm{m}$, this reduced distance corresponds to $\Delta=0.5, d$ becomes smaller than $R_{\mathrm{MP}}$ [see red MP of only $30 \mu \mathrm{m}$ radius in Fig. 15(a)]. Hence, the MP completely overshadows the tip. The consequence is that the MP replaces the tip and the electron emission becomes insignificant at the cathode because the MP aspect ratio is much lower than the tip. Therefore, the crash of large microparticles onto the cathode can reduce significantly the thermofield emission at the cathode surface. This could be an explanation for the cathode conditioning helping to increase the voltage held in vacuum without breakdown, experimentally observed when vacuum systems are submitted to high electric fields prior to their use [4].

\section{Heat transfer to the tip during the MP crash}

As a final analysis resulting from the present model, this part studies the thermal effect of the crash of a MP launched on the axis. As the previous subsection demonstrated an effect of dumping the field emission for large MP, by also the possibility of enhancing the electron emission current by the increase aspect ratio by the crash of NPs onto the tip apex, the tip by an increase of its temperature leading to higher thermoionic emission will be now examined.

Assuming that the kinetic energy of the MP is completely transformed into thermal energy, the thermal energy balance can be used to determine the effect of this heating on the tip.

Figure 16 represents the kinetic energy carried by MPs with respect to their radius when it crashes on the tallest tip $\left(H_{\text {tip }}=10 \mu \mathrm{m}\right)$ for a very low current $\left(\mathrm{I}=10^{-6} \mathrm{~A}\right)$. This energy has to be compared to the energy required to vaporize the emissive volume situated at the tip apex (Fig. 4). This vaporization energy is equal to the latent energy $\left(8 \times 10^{-7} \mathrm{~J}\right)$ whereas the energy to heat the tip apex up to the vaporization temperature $\left(5 \times 10^{-8} \mathrm{~J}\right)$ is at least 1 order of magnitude lower, and therefore negligible. Considering this additional energy required to vaporize the emissive area (tip apex), for the highest tip $\left(H_{\text {tip }}=10 \mu \mathrm{m}\right)$ a threshold value appears of $10^{-10} \mathrm{~J}$ (red line Fig. 16). It represents less than $0.02 \%$ of the total volume of the tip, for the elliptical case considered here. Hence, if all the kinetic energy of the MP is transformed into thermal energy, a MP of $1 \mu \mathrm{m}$ radius could vaporize the electron emission area. For larger particles (10 $\mu$ m radius), more than $1 \%$ of the total volume of the tip will be vaporized during the crash. Moreover, the vaporization could be underestimated because secondary emission caused by the MP can also enhance the Joule effect inside the tip.

In the case of the smallest tip $\left(H_{\text {tip }}=0.2 \mu \mathrm{m}\right)$, this threshold energy is much lower, $\sim 5 \times 10^{-12} \mathrm{~J}$ (magenta line in Fig. 16), corresponding to the energy carried by small MP, of $100 \mathrm{~nm}$ radius and the small tip is completely vaporized.

The vaporization of the emission area flattens the tip apex and decreases the emission area. This effect takes part on the process of the electrodes conditioning acting in a

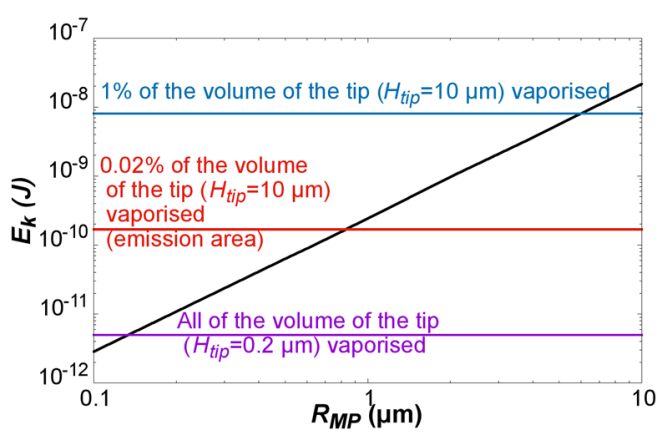

FIG. 16. Kinetic energy of the MP during the crash with the tip versus the $\mathrm{MP}$ radius, for $\mathrm{I}=0.1 \mu \mathrm{A}, \mathrm{V}_{\text {app }}=25 \mathrm{kV}$, and $\mathrm{d}=0.5 \mathrm{~mm}$. 
similar way with the reduction of field emission found in the previous subsection.

\section{CONCLUSIONS}

Let us summarize below the conclusions drawn out from this theoretical study of the transit of microparticles released from the anode and traveling between the electrodes under high voltage and in vacuum.

The study of the interaction of the MP with the electron beam has shown that the cross section of the MP with the energetic electrons is almost equal to the area of the MP. The higher the primary electron velocity (i.e., relativistic electrons) is, the closer the interaction cross section to the MP section. Consequently, the presence of the MP does not affect the primary electron beam passing around the MP.

However, this interaction has important consequences on charge variation caused by secondary electron emission due to energetic electron irradiation of the MP and by electron capture inside the MP (stopping power). The latter induces the clump heat-up causing the evaporation of the microparticle and consequently a significant change in size.

All these phenomena involve different transit regimes in the interelectrode gap. Four regimes have been clearly identified.

(1) The MP arrives at the cathode for very small currents, called one way path.

(2) The MP collects enough electrons to become negative and returns to the anode, called anode return.

(3) The MP size decreases because the electron current heats up the MP. However, it does not trap enough electrons to come back to the anode. During the transition with the previous regime, oscillations can take place and the MPs move forth and back until they reach one of the electrodes, mainly the cathode.

(4) For very high currents, complete vaporization of the MP occurs in the interelectrode gap.

The study of the trajectories of the MPs shows that the electric field at the tip apex has no influence on the clump trajectory in the interelectrode gap because the microparticle inertia is too large.

But the MP size plays on electron emission. Large MPs can cover the tip and cancel the thermofield electron emission or vaporize the tip by heat transfer. These phenomena lead to the system conditioning reducing the dark current, as experimentally found.

The space charge effect in the interelectrode gap created by the interaction of the MP with the tip will be the topic of future work.

\section{ACKNOWLEDGMENTS}

This work was supported by the French National Research Agency (ANR) as part of the program "High Voltage holding In Vacuum (HVIV)" (Reference No. ANR12-BS09-013).
[1] R. S. Hemsworth, H. Decamps, J. Graceffa, B. Schunke, M. Tanaka, M. Dremel, A. Tanga, H. P. L. De Esch, F. Geli, J. Milnes, T. Inoue, D. Marcuzzi, P. Sonato, and P. Zaccaria, Status of the ITER heating neutral beam system , Nucl. Fusion 49, 045006 (2009).

[2] Simonin, H. de Esch, L. Doceul, L. Christin, F. Faisse, and F. Villecroze, Conceptual design of a high-voltage compact bushing for application to future N-NBI systems of fusion reactors, Fusion Eng. Des. 88, 1 (2013).

[3] A. Revel, S. Mochalskyy, L. Caillault, A. Lifschitz, and T. Minea, Transport of realistic beams in ITER neutral beam injector accelerator, Nucl. Fusion 53, 073027 (2013).

[4] R. V. Latham, High Voltage Vacuum Insulation, The Physical Basis (Academic Press, New York, 1981).

[5] G. N. Fursey, Field emission and vacuum breakdown, IEEE Trans. Electr. Insul. EI-20, 659 (1985).

[6] G. A. Mesyats and D. I. Proskurosvsky, Pulsed Electrical Discharge in Vacuum (Springer-Verlag, Berlin, 1984).

[7] W. P. Dyke and J. K. Trolan, Field emission: Large current densities, space charge, and the vacuum arc, Phys. Rev. 89, 799 (1953).

[8] P. H. Devine, Thermoelectric phenomena associated with electron-field emission, J. Appl. Phys. 33, 582 (1962).

[9] D. Alpert, D. Lee, E. M. Lyman, and H. E. Tomaschke, Effect of gas pressure on electrical breakdown and field emission, J. Appl. Phys. 38, 880 (1967).

[10] W. B. Nottingham, Remarks on energy losses attending thermionic emission of electrons from metals, Phys. Rev. 59, 906 (1941).

[11] J. R. Haase and D. B. Go, Analysis of thermionic and thermo-field emission in microscale gas discharges, J. Phys. D 49, 055206 (2016).

[12] A. V. Phelps and Z. L. J. Petrovic, Cold-cathode discharges and breakdown in argon: surface and gas phase production of secondary electrons, Plasma Sources Sci. Technol. 8, R21 (1999).

[13] A. Descoeudres, Y. Levinsen, S. Calatroni, M. Taborelli, and W. Wuensch, Investigation of the dc vacuum breakdown mechanism, Phys. Rev. ST Accel. Beams 12, 092001 (2009).

[14] K. Nordlund and F. Djurabekova, Defect model for the dependence of breakdown rate on external electric fields, Phys. Rev. ST Accel. Beams 15, 071002 (2012).

[15] A. Gohier, M. A. Djouadi, M. Dubosc, A. Granier, T. M. Minea, L. Sirghi, F. Rossi, P. Paredez, and F. Alvarez, Single- and few-walled carbon nanotubes grown at temperatures as low as $450^{\circ} \mathrm{C}$ : electrical and field emission characterization, J. Nanosci. Nanotechnol. 7, 3350 (2007).

[16] R. H. Fowler and L. Nordheim, Electron emission in intense electric fields, Proc. R. Soc. A 119, 173 (1928).

[17] J. G. Trump and R. J. Van de Graaf, The insulation of high voltages in vacuum, J. Appl. Phys. 18, 327 (1947).

[18] L. Cranberg, The initiation of electrical breakdown in vacuum, J. Appl. Phys. 23, 518 (1952).

[19] A. Maitland, New derivation of the vacuum breakdown equation relating breakdown voltage and electrode separation, J. Appl. Phys. 32, 2399 (1961).

[20] N. Pilan, P. Veltri, and A. De Lorenzi, Voltage holding prediction in multi electrode-multi voltage systems 
insulated in vacuum, IEEE Trans. Dielectr. Electr. Insul. 18, 553 (2011).

[21] G. P. Beukema Electrical breakdown properties of stainless steel and titanium electrodes in ultra-high vacuum, Physica (Amsterdam) 103B+C, 397 (1981).

[22] G. A. Farrall, Cranberg hypothesis of vacuum breakdown as applied to impulse voltages, J. Appl. Phys. 33, 96 (1962).

[23] I. N. Slivkov, Mechanism for electrical discharge in vacuum, Sov. Phys. Technol. 2, 1928 (1957).

[24] M. M. Menon and K. D. Srivastava, Detection and identification of microparticles occurring in a high-voltage vacuum-insulated gap using electron optical methods, J. Appl. Phys. 45, 2094 (1974).

[25] D. A. Eastham and P. A. Chatterton, The dectection of microparticle-induced breakdowns using a twin-beam laser scattering system, IEEE Trans. Electr. Insul. EI-18, 209 (1983).

[26] D. K. Davies and M. A. Biondi, Mechanism of dc electrical breakdown between extended electrodes in vacuum, J. Appl. Phys. 42, 3089 (1971).

[27] A. Chatterton, M. M. Menon, and K. D. Srivastava, Processes involved in the triggering of vacuum breakdown by low-velocity microparticles, J. Appl. Phys. 43, 4536 (1972).

[28] E. Pozdeyev, Ion trapping and cathode bombardment by trapped ions in dc photoguns, Phys. Rev. ST Accel. Beams 10, 083501 (2007).

[29] F. Zimmermann, J. T. Seeman, M. Zolotorev, and W. stoeffl, in Proceedings of the Particle Accelerator Conference, Dallas, TX, 1995 (IEEE, New York, 1995).

[30] F. Zimmermann, M. Giovannozzi, and A. Xagkoni, in Proceedings of the International Particle Accelerator Conference, Kyoto, Japan (ICR, Kyoto, 2010).

[31] S. Heifets, Q. Qin, and M. Zolotorev, Life of the dust macroparticles in storage rings, Phys. Rev. ST Accel. Beams 8, 061002 (2005).

[32] S. Rowan, A. Apollonio, B. Auchmann, A. Lechner, O. Picha, W. Riegler, H. Schindler, R. Schmidt, and F. Zimmermann, in Proceedings of IPAC2015, Richmond, USA (JACoW, Geneva, 2015).

[33] D. K. Davies and M. A. Biondi, Emission of electrode vapor resonance radiation at the onset of dc breakdown in vacuum, J Appl. Phys. 48, 4229 (1977).

[34] R. Miller, Y. Y. Lau, and J. H. Booske, Schottky's conjecture on multiplication of field enhancement factors, J. Appl. Phys. 106, 104903 (2009).

[35] K. L. Jensen, D. A. Shiffler, J. R. Harris, and J. J. Petillo, Schottky's conjecture, field emitters, and the point charge model, AIP Adv. 6, 065005 (2016).

[36] W. T. Diamond, New perspectives in vacuum high voltage insulation. I. The transition to field emission, J. Vac. Sci. Technol. A 16, 707 (1998).

[37] N. Nishimori, R. Nagai, S. Matsuba, R. Hajima, M. Yamamoto, Y. Honda, T. Miyajima, H. Iijima, M. Kuriki, and M. Kuwahara, Experimental investigation of an optimum configuration for a high-voltage photoemission gun for operation at $\geq 500 \mathrm{kV}$, Phys. Rev. ST Accel. Beams 17, 053401 (2014).
[38] D. K. Davies and M. A. Biondi, The effect of electrode temperature on vacuum electrical breakdown between plane-parallel copper electrodes, J. Appl. Phys. 39, 2979 (1968).

[39] E. L. Murphy and R. H. Good, Jr., Thermionic emission, field emission, and the transition region, Phys. Rev. 102, 1464 (1956).

[40] M. G. Ancona, Thermomechanical analysis of failure of metal field emitters, J. Vac. Sci. Technol. B 13, 2206 (1995).

[41] A. C. Keser, T. M. Antonsen, G. S. Nusinovich, D. G. Kashyn, and K. L. Jensen, Heating of microprotrusions in accelerating structures, Phys. Rev. ST Accel. Beams 16, 092001 (2013).

[42] B. Seznec, Ph. Dessante, L. Caillault, J. L. Babigeon, Ph. Teste, and T. Minea, Controlled electron emission and vacuum breakdown with nanosecond pulses, J. Phys. D 49, 235502 (2016).

[43] http://www.comsol.com.

[44] R. G. Forbes and J. H. B. Deane, Reformulation of the standard theory of Fowler-Nordheim tunnelling and cold field electron emission, Proc. R. Soc. A 463, 2907 (2007).

[45] A. Fischer, M. S. Mousa, and R. G. Forbes, Influence of barrier form on Fowler-Nordheim plot analysis, J. Vac. Sci. Technol. B 31, 032201 (2013).

[46] A. Kyritsakis, G. C. Kokkorakis, J.P. Xanthakis, T. L. Kirk, and D. Pescia, Self focusing of field emitted electrons at an ellipsoidal tip, Appl. Phys. Lett. 97, 023104 (2010).

[47] R. E. Hurley and T. M. Parnell, Field emission from metal particles in a vacuum gap, J. Phys. D: Appl. Phys. 2, 881 (1969).

[48] J. H. Jeans, The Mathematical Theory of Electricity and Magnetism (Cambridge Press, London, 1958).

[49] F. F. Chen, J. D. Evans, and W. Zawalski, Electric Probes in Plasma Diagnostic Techniques, edited by R. H. Huddlestone and S. L. Leornard (Academic Press, New York, 1965), Chap. 4, p. 113.

[50] http://physics.nist.gov/PhysRefData/Star/Text/ESTAR.html.

[51] H. A. Bethe, Zur Theorie des Durchgangs schneller Korpuskularstrahlen durch Materie, Ann. Phys. (Paris) 397, 325 (1930).

[52] H. A. Bethe, Bremsformel für Elektronen relativistischer Geschwindigkeit, Z. Phys. 76, 293 (1932).

[53] ICRU Report No. 37 (1984).

[54] A. J. Dekker, Secondary electron emission, Solid State Phys. 6, 251 (1958).

[55] L. Vignitchouk, P. Tolias, and S. Ratynskaia, Dust-wall and dust-plasma interaction in the MIGRAINe code, Plasma Phys. Control. Fusion 56, 095005 (2014).

[56] V. W. Chow, D. A. Mendis, and M. Rosenberg, Secondary emission from small dust grains at high electron energies, IEEE Trans. Plasma Sci. 22, 179 (1994).

[57] D. R. Lide, CRC Handbook of Chemistry and Physics (CRC Press, Boca Raton, FL, 2005).

[58] See Supplemental Material at http://link.aps.org/ supplemental/10.1103/PhysRevAccelBeams.20.073501 for a description of the different regimes of the MP of a test 
emission current. Animations of regimes "One way", "Anode return", "Oscillation" and "Evaporation" have been represented.

[59] R. V. Latham, A. S. Brah, K. Fok, and M. O. Woods, Semielastic impact studies of carbonyl iron microspheres on diamond-polished metal targets (relevant to interelectrode bouncing of charged microparticles in a high-voltage vacuum gap), J. Phys. D 10, 139 (1977).

[60] Y. Tanimoto, T. Honda, and S. Sakanaka, Experimental demonstration and visual observation of dust trapping in an electron storage ring, Phys. Rev. ST Accel. Beams 12, 110702 (2009). 